\title{
ESCUELA DE CAMPO DE AGRICULTORES (ECAS) PARA CAPACITAR EN EL USO DE PLAGUICIDAS A LOS AGRICULTORES DE JESÚS DEL VALLE, HUARAL - LIMA
}

\author{
FARMER FIELD SCHOOL (FFS) TO TRAIN IN THE USE OF PESTICIDES TO FARMERS \\ OF JESUS DEL VALLE, HUARAL - LIMA
}

${ }^{1}$ Ana M. Viera Y. y ${ }^{2}$ Susana P. Rodríguez Q.

\begin{abstract}
Resumen
Se evaluó la metodología de Escuelas de Campo de Agricultores - ECA como un método apropiado para capacitar a los agricultores y contribuir en el conocimiento del uso y manejo correcto de plaguicidas, identificando las fuentes de información sobre este tema e identificando a los actores que influyen en la toma de decisiones de aplicación y uso de estos productos entre los agricultores. Se capacitó a los agricultores durante un ciclo productivo del cultivo de algodonero en su manejo agronómico y en el uso y manejo correcto de plaguicidas. Culminada la ECA, los agricultores fueron entrevistados en sus conocimientos sobre estos temas y obtuvieron un $89.4 \%$ de aciertos en sus respuestas, respecto a un $60 \%$ de aciertos de los agricultores que no fueron capacitados en la ECA.El 53.3\% de agricultores capacitados en la ECA y el $66.7 \%$ de agricultores que no participaron en una ECA, ambos señalan como principal fuente de información e influencia para decidir usar plaguicidas, al personal técnico de las tiendas de agroquímicos. Se concluyó que, la metodología de Escuelas de Campo de Agricultores (ECAs) es apropiada para capacitar a los agricultores en el "uso y manejo correcto de plaguicidas" en la medida que se trate el tema asociado al cultivo y a los problemas de manejo de su interés.
\end{abstract}

Palabras clave: Escuela de Campo de Agricultores, metodología de capacitación, uso correcto de plaguicidas.

\begin{abstract}
Methodology of Farmer Field Schools - FFS as a appropriate method to train farmers and contribute to the knowledge of the proper use and handling of pesticides, identifying the sources of information on this topic and identifying the actors that influence the decision-making application and use of these products among farmers. Were trained farmers for a crop production cycle of cotton in their agronomic management and the proper use and handling of pesticides. The completion of the FFS, farmers were interviewed in their knowledge of these issues and achieved $89.4 \%$ correct in their answers, about a $60 \%$ hit farmers who were not trained in the FFS. The FFS consider the management and solution of the problems that had arisen, always relating them to the study topic. 53.3\% of farmers trained in FFS and $66.7 \%$ of farmers who did not participate in a FFS, both designated as primary source of information and influence to decide to use pesticides, technical staff from the shops of agrochemicals. It was concluded that the methodology of Farmer Field Schools (FFS) is appropriate to train farmers in the "proper use and handling of pesticides" as the theme associated with the cultivation and management problems your question interest.
\end{abstract}

Key words: Farmer Field School, training methodology, proper use of pesticides.

\section{Introducción}

El manejo y uso de plaguicidas agrícolas es muy deficiente entre los pequeños agricultores de la Costa Central del Perú. La mayoría de ellos hacen un uso indiscriminado sin tomar en cuenta la protección personal, ni el cuidado del ambiente. Un $90 \%$ no puede reconocer la toxicidad de los plaguicidas agrícolas mediante la etiqueta del envase y el $95 \%$ no sabe cuáles son los primeros auxilios que se debe brindar a un intoxicado (PROTEC, 2007).

Hoy en día no se cuenta con un registro de casos de intoxicaciones y muertes por el uso de plaguicidas, debido a que aún no se ha implementado un sistema nacional que se encargue de recopilar este tipo de información, y a la vez la limitada capacitación de los profesionales de la salud en el registro, atención y diferenciación de casos de intoxicaciones por plaguicidas, que a veces pueden ser atribuidos a otras causas. A pesar de ello, algunas instituciones, como la Red de Acción en Agricultura Alternativa - RAAA, realizó algunos estudios sobre los efectos que tienen los plaguicidas en la salud de varones, mujeres y niños, tanto sus efectos agudos, causantes de muertes o enfermedades graves, como crónicos, en 
comunidades y/o localidades con gran actividad agrícola donde el uso de plaguicidas es de manera indiscriminada, y donde los síntomas por intoxicaciones pueden pasar desapercibidos. Los resultados de estos reportes dieron una clara evidencia del efecto negativo a la salud que vienen sufriendo los trabajadores del campo por el uso inadecuado de estos productos tóxicos.

El desconocimiento por parte de los agricultores del riesgo de intoxicaciones al que se expone, sumado a la escasa información con la que cuentan sobre las medidas preventivas en cuanto a la manipulación y almacenamiento de plaguicidas, genera una importante demanda de capacitación. Esta necesidad ha llevado a algunos esfuerzos aislados, pero con resultados poco alentadores.

El Comité para la Protección de Cultivos - PROTEC, de la Cámara de Comercio de Lima, y el Consejo de Gestión de la Calidad y Buenas Prácticas Agrícolas - BPA del Valle Huaral-Chancay, conformado por las Comisiones de Regantes de la jurisdicción, el SENASA, el INIA, entre otras instituciones, abordaron de forma directa el problema de la escasa capacitación, y usó la metodología de Escuelas de Campo de Agricultores ECA para capacitar a los pequeños agricultores en el "uso y manejo correcto de plaguicidas".

El presente estudio evaluó la metodología de Escuelas de Campo y su contribución para incrementar el conocimiento de los agricultores de Jesús del Valle, Huaral en el uso y manejo correcto de plaguicidas, identificando las fuentes de información y su influencia en la toma de decisiones de aplicación y uso de estos productos. La premisa sometida a prueba es que la metodología de capacitación de Escuelas de Campo de Agricultores (ECAs) es apropiada para capacitar agricultores en el "uso y manejo correcto de plaguicidas".

\section{Materiales y métodos}

El presente estudio se desarrolló en la localidad de Huarangal, perteneciente al distrito de Jesús del Valle, provincia de Huaral, Lima, Perú, ubicada a $1 \mathrm{Km}$. al sur de Huaral donde la actividad productiva más importante es la agricultura, destacando cultivos como tomate, papa, camote, fresas, entre otros, y cultivos industriales como maíz amarillo duro y algodonero, todos orientados al mercado nacional.

Se desarrolló una ECA en el cultivo de algodonero en la campaña 2005-2006 para capacitar a los agricultores en el tema de manejo y uso correcto de plaguicidas, durante y después de la cual se realizó la evaluación.

La población quedó definida por todos los agricultores de Jesús del Valle registrados en la Junta de Usuarios del Distrito de Riego del Valle de Chancay-Huaral, estos ascienden a 592. El tamaño de la muestra quedó definido por la siguiente fórmula (Hernández, et al., 1998):

$$
\mathrm{n}=\underline{\underline{\mathrm{n}^{\prime}}} \underset{\overline{1+\mathrm{n}^{\prime} / \mathrm{N}}}{\mathrm{n}^{\prime}=\frac{\mathrm{S}^{2}}{\mathrm{~V}^{2}}}
$$

donde: $\quad \mathrm{n}=$ tamaño de la muestra

$$
\mathrm{S}^{2}=\mathrm{p}(1-\mathrm{p})
$$$$
\mathrm{p}=\text { probabilidad de acierto (se asume } 0.5 \text { ) }
$$$$
\mathrm{V}^{2}=(\mathrm{se})^{2}
$$$$
\text { se }=\text { error estándar (0.09) }
$$$$
\mathrm{N}=\text { población }
$$

operando: $\quad \mathrm{n}=29$

Se consideró un error de $9 \%$ ya que la metodología de las ECAs trabaja la actitud de los participantes, por lo tanto hay muchos aspectos no medibles y se puede aceptar un error alto. Considerando este error del $9 \%$ se determinó una muestra de 29 agricultores, pero con fines de poder hacer una comparación entre 2 grupos de participantes y no participantes a la Escuela de Campo, la muestra se completó a 30 agricultores.

La muestra de 30 agricultores se dividió en 2 grupos de 15 cada uno a fin de poder hacer la comparación entre, un grupo que está conformado por los participantes de la ECA de Huarangal y el otro grupo "testigo" que no recibió esta capacitación, asegurándose previamente que ninguno recibió una capacitación especial en este tema y su conocimiento era homogéneo. Los participantes eligieron trabajar la ECA en el cultivo de algodonero, por ser el cultivo de mayor importancia e interés para los agricultores. La ECA registró asistencia permanente de sus participantes y un número equitativo de participantes hombres y mujeres. Los 15 agricultores del grupo "testigo" que no participaron de una ECA fueron escogidos al azar, siempre que pertenecieran al distrito de Jesús del Valle. Esto con el fin de que los resultados de la investigación puedan ser comparables y tengan validez.

La ECA, en su primera sesión programó conjuntamente con los agricultores el número de sesiones y temas de capacitación que fueron 12 , cada sesión consideró temas de manejo del cultivo y enfatizó en el buen manejo de los plaguicidas que se programaron, entre herbicidas e insecticidas, no fue necesario el uso de fungicidas u otro tipo de plaguicida agrícola para este cultivo. Cada sesión de capacitación se desarrolló en una parcela de aprendizaje seleccionada con los agricultores y consideró el análisis agroecológico, el tema de capacitación y actividades programadas, los compromisos para la siguiente sesión, la evaluación de la sesión del día, así como el espacio de refrigerio u almuerzo según se dieron las jornadas de campo, todo como lo indica la metodología. Las sesiones fueron grupales y de carácter constructivo, es decir que el aprendizaje se dio tomando decisiones de manejo previa evaluación y análisis del cultivo, en el quehacer de cada sesión. Los temas programados para cada sesión fueron: 1. la preparación del campo, 2. la siembra, 3. la fertilización, 4. riego, 5. Identificación de plagas y controladores biológicos, 6. Evaluación de campo, 7. Lectura de etiqueta, 8. Transporte y almacenamiento, 9. Preparación de la mezcla y equipos, 10. Protección durante la aplicación y post aplicación, 11. Cosecha, 12. La prueba de la chacra. 
Las variables independientes consideradas fueron: sexo, edad, manejo del predio, área que posee, y cultivos que maneja. Para medir el aprendizaje obtenido en el tema del "manejo y uso correcto de plaguicidas agrícolas" se plantearon como variables dependientes: evaluación previa del campo, lectura de etiqueta, transporte y almacenamiento, preparación de la mezcla y equipos, protección durante la aplicación, y post-aplicación.

Se realizaron algunas entrevistas y se aplicó una encuesta semi-estructurada a toda la muestra de agricultores, para poder establecer las diferencias y si estas son significativas como para ser atribuidas a la participación en una ECA.

Cada variable dependiente fue tomada en el cuestionario de la encuesta como ítem. Estos ítems estaban conformados por preguntas que, según si eran respondidas correcta o incorrectamente, obtenían un puntaje que varía del 1 al 5, siendo 1 el valor asignado al menor conocimiento y 5 al de mayor conocimiento. La asignación de los puntajes se dio en función al cumplimiento de lo siguiente:

Evaluación previa del campo: ¿Conoce las principales plagas que atacan a sus cultivos? ¿Posee un sistema de evaluación y monitoreo de las plagas en sus campos? ¿Lleva un registro de la fecha de evaluación, cultivo y su estado fenológico, e incidencia de la plaga? ¿Conoce otras alternativas (por lo menos 2), aparte del control químico, para controlar estas plagas? ¿Decide usar plaguicidas sólo luego de realizar esta evaluación? ¿Recibe asistencia técnica en este proceso?

Lectura de etiqueta: ¿El producto que selecciona es específico para la plaga y el cultivo? ¿Conoce el significado de las bandas de color de toxicidad en el envase? ¿Puede mencionar algunas medidas de seguridad (como mínimo 3) que aparecen en la etiqueta de un plaguicida? ¿Conoce y entiende el "período de carencia" de un producto (aunque no lo asocie directamente con este término)? ¿El producto que elige está registrado en SENASA? ¿Compró en tiendas comerciales de confianza?

Transporte y almacenamiento: ¿Se puede almacenar el pesticida junto con alimentos, semilla, ropa de aplicación $\mathrm{u}$ otros productos? ¿El transporte de plaguicidas se realiza separado de alimentos, semillas, personas y animales? ¿Mantiene el producto en su envase original? ¿Qué características debe tener el almacén donde se guarden los pesticidas? ¿Qué debemos encontrar en él? ¿El almacenamiento de pesticidas se da en un cuarto o compartimiento donde se guardan ropa de aplicación, semillas, o fertilizantes? ¿El almacén o compartimiento está alejado de los niños y se mantiene bajo llave? ¿Este cuenta con ventilación adecuada y señalización de peligro?

Preparación de la mezcla y equipos: ¿Conoce las dosis y recomendaciones básicas de uso para los productos que usa? ¿Entiende las recomendaciones en caso de intoxicaciones y primeros auxilios? ¿Se protege a la hora de preparar la mezcla usando como mínimo: guantes, pañuelo o mascarilla y camisa manga larga? ¿Revisa previamente sus equipos para la aplicación poniendo énfasis en boquillas, mangueras y filtros? ¿Hace la "calibración" de sus equipos (aunque no lo asocie directamente con este término)?

Protección durante la aplicación: Se protege a la hora de realizar la aplicación usando como mínimo protección para el tronco, brazos, manos, rostro, y pies (al menos pantalón y camisa impermeable de manga larga, guantes, mascarilla, mandil o bolsa de fertilizante y botas)? ¿Al momento de la aplicación quiénes pueden estar presentes en el campo (no se encuentran niños, animales, ni otras personas en el campo)? ¿Qué cosas no debe hacer cuando aplica (no aplica en horas de excesivo calor, contra del viento, cuando come, cuando bebe o cuando fuma)? ¿Lleva un registro de las aplicaciones que hace (anotando como mínimo: cultivo y variedad, fecha de aplicación, nombre del aplicador, plaga/enfermedad/maleza controlada, nombre del plaguicida y su ingrediente activo, dosis, período de carencia)?

Post-aplicación: ¿Si sobra mezcla hace un repase a los bordes del campo o zonas de barbecho, o lo arroja a la acequia? ¿Somete los envases vacíos a un triple lavado y luego los destruye y almacena en un lugar aparte? ¿Cómo se realiza el aseo personal y de la ropa del aplicador (lava los equipos usados con agua limpia, no arroja esta agua a las acequias, inmediatamente ha terminado su trabajo se baña con agua limpia y jabón, la ropa usada en la aplicación se lava de manera separada de la ropa del resto de la familia)?

La investigación evaluó el aprendizaje adquirido para cada variable dependiente de los dos grupos de agricultores, que participaron y no participaron en la ECA; y se diferenciaron entre sí de manera significativa respecto a sus medias usando la prueba " $\mathrm{t}$ ".

También se recogió información sobre las fuentes de información y su influencia en la toma de decisiones de aplicación y uso de los productos plaguicidas mediante entrevistas.

\section{Resultados y discusión}

De acuerdo al sexo de los participantes de la ECA, el $73.3 \%$ fueron varones y el $26,7 \%$ fueron mujeres, del total de 15 . El rango de edades entre los participantes, un $20 \%$ tenía menos de 45 años de edad, $53.3 \%$ entre 45 y 60 años de edad, y $26.7 \%$ con más de 60 años de edad. El área del predio de los participantes fue de $13.3 \%$ entre 1 a 3 has, $46.7 \%$ entre 3 y 5 has, de 5 a 10 has $26.7 \%$ y el $13.3 \%$ de los participantes de la ECA no posee tierras y son trabajadores de campo que ofrecen su mano de obra o que solicitan alquiler de tierras para producir. La mitad de los agricultores manejan 5 has o menos y los cultivos más sembrados son algodón 16, papa 15 y maíz 13, que suelen producir año a año. Otros cultivos de rotación entre campañas son leguminosas 10 (arveja y frijol), camote 4 , y frutales 4 (palto, lúcumo).

Los resultados del conocimiento adquirido por los agricultores en el uso correcto de plaguicidas según el 
rango de puntaje del 1 al 5, refererido a los temas de: "evaluación previa del campo", "lectura de etiqueta", "transporte y almacenamiento", "preparación de la mezcla y equipos", "protección durante la aplicación", y “post-aplicación”, se muestran en los puntajes obtenidos en las Fig. 1 y 2, tanto para los que participaron de una ECA como los que no tuvieron ninguna participación.
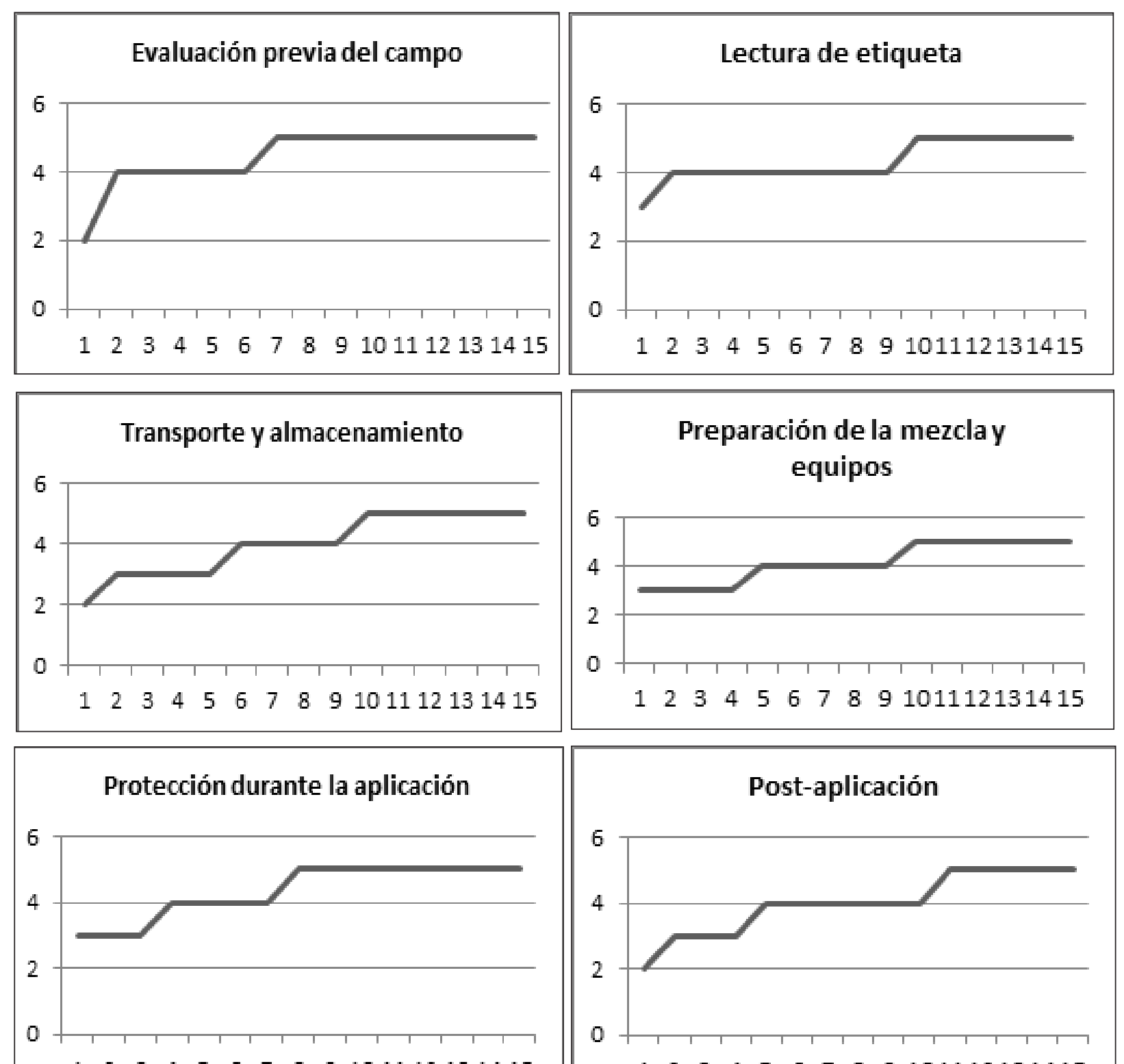

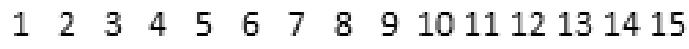

$\begin{array}{llllllllll}1 & 2 & 3 & 4 & 5 & 6 & 7 & 8 & 9 & 101112131415\end{array}$

Figura 1. Puntajes obtenidos por los 15 agricultores de la ECA de Huarangal en Jesús del Valle. 

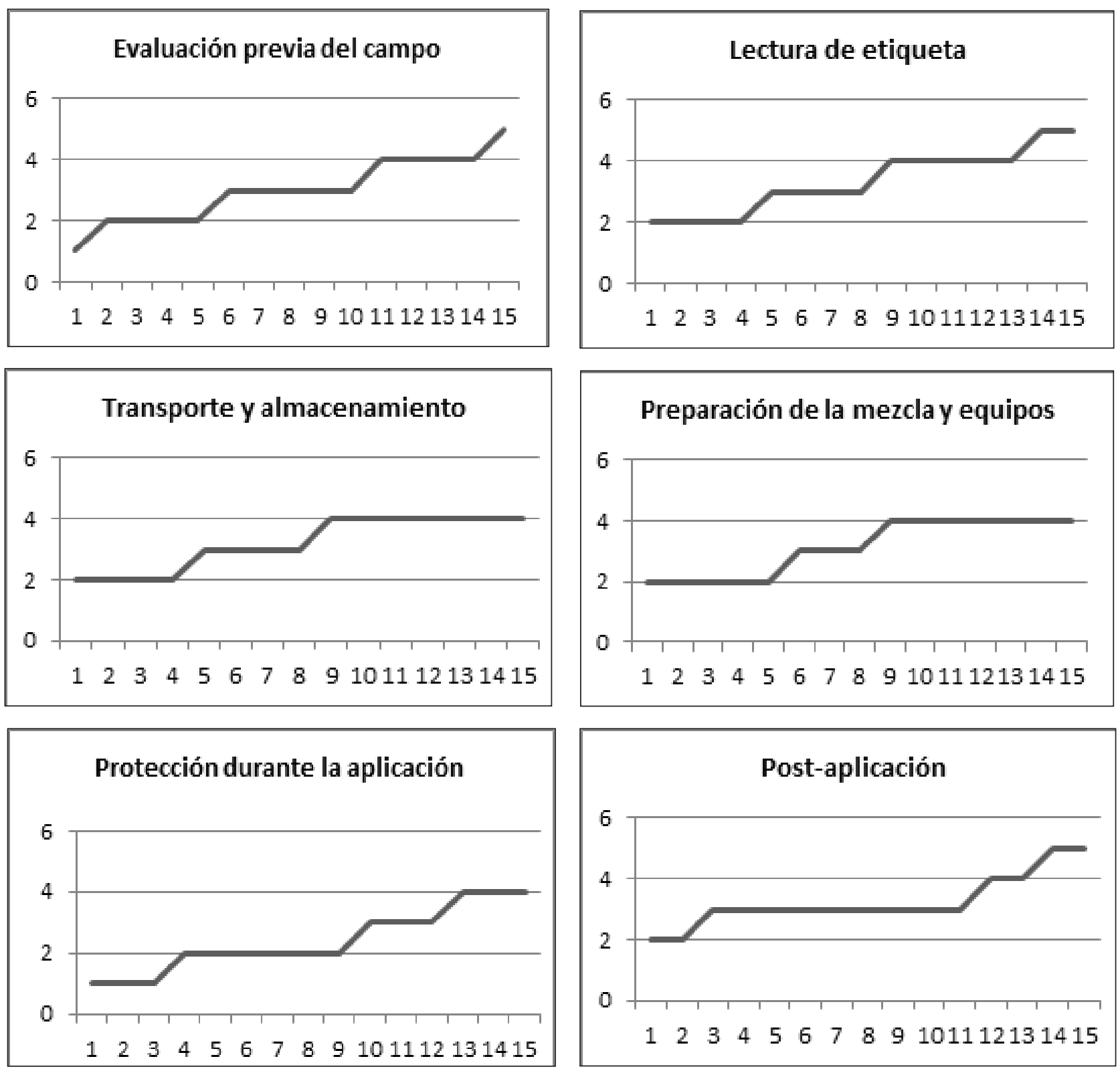

Figura 2. Puntajes obtenidos por los 15 agricultores que no participaron en la ECA de Huarangal en Jesús del Valle.

Según los resultados de las Fig. 1 y 2, para el tema de "evaluación previa del campo", los agricultores que participaron de la ECA sumaron un puntaje total de $67(89.4 \%)$ en este subtema, mientras que los que no participaron un total de $45(60 \%)$ puntos. En promedio, los participantes de la ECA obtuvieron 4.47 puntos y 3 puntos los no participantes de la ECA. Es decir, los agricultores que participaron en la ECA ahora poseen un $29.4 \%$ más de conocimiento acerca de la "evaluación previa del campo", respecto a los que no participaron

Este alto porcentaje de acierto respecto a la "evaluación de campo" se observa también en el estudio realizado por Groeneweg, Versteeg y Chávez (2004) que evaluó este aspecto en agricultores que participaron en varias ECAs en diferentes departamentos obteniendo un porcentaje cercano al $80 \%$.
Para evaluar si existe una diferencia significativa entre los puntajes de agricultores que participaron de la ECA y los que no lo hicieron, respecto a la "evaluación previa del campo", se aplicó la prueba "t" arrojando los siguientes resultados:

Tabla 1. Puntajes registrados para los participantes a la ECA en el tema "evaluación previa del campo".

\begin{tabular}{cccc}
\hline $\begin{array}{c}\text { Puntajes } \\
\text { registrados }\end{array}$ & Frecuencias & $\begin{array}{c}\text { Porcentajes } \\
\text { relativos }\end{array}$ & $\begin{array}{c}\text { Porcentajes } \\
\text { acumulados }\end{array}$ \\
\hline 5 & 9 & 60 & 60 \\
4 & 5 & 33.3 & 93.3 \\
2 & 1 & 6.7 & 100 \\
\hline Total & 15 & 100 & \\
\hline
\end{tabular}


Según la tabla 1 , el puntaje que más se repitió fue 5 . Con respecto a la media, el $50 \%$ de los agricultores entrevistados obtuvo un puntaje por encima de 5 y el $50 \%$ restante obtuvo un puntaje por debajo de este valor. En promedio, el puntaje obtenido es de 4.47. Asimismo, se desvían de 4.47 (en promedio) 0.79 puntos.

Tabla 2. Puntajes registrados para los no participantes a la ECA en el tema "evaluación previa del campo".

\begin{tabular}{cccc}
\hline $\begin{array}{c}\text { Puntajes } \\
\text { registrados }\end{array}$ & Frecuencias & $\begin{array}{c}\text { Porcentajes } \\
\text { relativos }\end{array}$ & $\begin{array}{c}\text { Porcentajes } \\
\text { acumulados }\end{array}$ \\
\hline 5 & 1 & 6.7 & 6.7 \\
4 & 4 & 26.7 & 33.4 \\
3 & 5 & 33.2 & 66.6 \\
2 & 4 & 26.7 & 93.3 \\
1 & 1 & 6.7 & 100 \\
\hline Total & 15 & 100 & \\
\hline
\end{tabular}

Según la tabla 2, El 50\% de los agricultores entrevistados obtuvo un puntaje por encima de 3 y el $50 \%$ restante obtuvo un puntaje por debajo de este valor.

Los agricultores de Jesús del Valle que han participado en la ECA poseen mayor información y conocimiento acerca de la "evaluación previa del campo" que los agricultores que no participaron en un nivel de confianza del 99\%.

Según las figuras 1 y 2, para el tema de "lectura de etiqueta", los agricultores que participaron en la ECA sumaron un puntaje total de $65(86.6 \%)$ en este tema, mientras que los que no participaron un total de 50 (66.6\%) puntos. En promedio se puede ver que los participantes de a la ECA obtuvieron 4.33 puntos y 3.33 los no participantes. Es decir, los agricultores que participaron en la ECA ahora poseen un $20 \%$ más de conocimiento acerca de la "lectura correcta de la etiqueta del producto", que los no participantes.

Para evaluar si existe una diferencia significativa entre los puntajes de agricultores que participaron en la ECA y los que no lo hicieron respecto a la "lectura de etiqueta", se aplicó la prueba " $t$ " arrojando los siguientes resultados:

Tabla 3. Puntajes registrados para los participantes de la ECA en el tema "lectura de etiqueta".

\begin{tabular}{cccc}
\hline $\begin{array}{c}\text { Puntajes } \\
\text { registrados }\end{array}$ & Frecuencias & $\begin{array}{c}\text { Porcentajes } \\
\text { relativos }\end{array}$ & $\begin{array}{c}\text { Porcentajes } \\
\text { acumulados }\end{array}$ \\
\hline 5 & 6 & 40 & 40 \\
4 & 8 & 53.3 & 93.3 \\
3 & 1 & 6.7 & 100 \\
\hline Total & 15 & 100 & \\
\hline
\end{tabular}

Según la tabla 3, el puntaje que más se repitió fue 4 . Con respecto a la media, el $50 \%$ de los agricultores entrevistados obtuvo un puntaje por encima de 4 y el $50 \%$ restante obtuvo un puntaje por debajo de este valor. En promedio, el puntaje obtenido es de 4.33. Asimismo, se desvían de 4.33 (en promedio) 0.6 puntos.

Según la tabla 4, el puntaje que más se repitió fue 4 . Con respecto a la media, el $50 \%$ de los agricultores entrevistados obtuvo un puntaje por encima de 3 y el $50 \%$ restante obtuvo un puntaje por debajo de este valor. En promedio, el puntaje obtenido es de 3.33. Asimismo, se desvía de 3.33 (en promedio) 1 punto.

Tabla 4. Puntajes registrados para los no participantes a la ECA en el tema "lectura de etiqueta".

\begin{tabular}{cccc}
\hline $\begin{array}{c}\text { Puntajes } \\
\text { registrados }\end{array}$ & Frecuencias & $\begin{array}{c}\text { Porcentajes } \\
\text { relativos }\end{array}$ & $\begin{array}{c}\text { Porcentajes } \\
\text { acumulados }\end{array}$ \\
\hline 5 & 2 & 13.4 & 13.4 \\
4 & 5 & 33.2 & 46.6 \\
3 & 4 & 26.7 & 73.3 \\
2 & 4 & 26.7 & 100 \\
\hline Total & 15 & 100 & \\
\hline
\end{tabular}

Los agricultores de Jesús del Valle que han participado en la ECA poseen mayor información y conocimiento acerca de la "lectura de etiqueta" que los agricultores que no participaron, en un nivel de confianza del 99\%.

Según las Fig. 1 y 2, los agricultores que participaron en la ECA sumaron un puntaje total de $60(80 \%)$ en el tema de "transporte y almacenamiento", mientras que los que no participaron un total de 48 (64\%). En promedio se puede ver que los participantes a la ECA obtuvieron 4 puntos en tanto, los que no participaron de la ECA obtuvieron un promedio de 3.2 puntos.

Es decir, los agricultores que participaron en la ECA ahora poseen un $16 \%$ más de conocimiento acerca del "transporte y almacenamiento correcto de los plaguicidas" respecto a los no participantes como puede observarse en la tabla 5

Tabla 5. Puntaje total, promedio y porcentaje de acierto en el tema "transporte y almacenamiento", entre agricultores participantes y no participantes en la ECA.

\begin{tabular}{cccc}
\hline & $\begin{array}{c}\text { Total } \\
\text { puntaje }\end{array}$ & Promedio & $\begin{array}{c}\% \\
\text { Acierto }\end{array}$ \\
\hline $\begin{array}{c}\text { Participantes de la ECA } \\
\begin{array}{c}\text { No participantes de la } \\
\text { ECA }\end{array}\end{array}$ & 60 & 4 & 80 \\
\hline
\end{tabular}

Para evaluar si existe una diferencia significativa entre los puntajes de agricultores que participaron en la ECA y los que no lo hicieron respecto a "transporte y almacenamiento de plaguicidas", se aplicó la prueba " $\mathrm{t}$ " arrojando los siguientes resultados: 
Tabla 6. Puntajes registrados para los participantes de la ECA en el tema "transporte y almacenamiento".

\begin{tabular}{cccc}
\hline $\begin{array}{c}\text { Puntajes } \\
\text { registrados }\end{array}$ & Frecuencias & $\begin{array}{c}\text { Porcentajes } \\
\text { relativos }\end{array}$ & $\begin{array}{c}\text { Porcentajes } \\
\text { acumulados }\end{array}$ \\
\hline 5 & 6 & 39.9 & 39.9 \\
4 & 4 & 26.7 & 66.6 \\
3 & 4 & 26.7 & 93.3 \\
2 & 1 & 6.7 & 100 \\
\hline Total & 15 & 100 & \\
\hline
\end{tabular}

Según la tabla 6 , el puntaje que más se repitió fue 5 . Con respecto a la media, el $50 \%$ de los agricultores entrevistados obtuvo un puntaje por encima de 4 y el $50 \%$ restante obtuvo un puntaje por debajo de este valor. En promedio, el puntaje obtenido es de 4. Asimismo, se desvía de 4 (en promedio) 0.97 puntos.

Tabla 7. Puntajes registrados para los no participantes de la ECA en el tema "transporte y almacenamiento".

\begin{tabular}{cccc}
\hline $\begin{array}{c}\text { Puntajes } \\
\text { registrados }\end{array}$ & Frecuencias & $\begin{array}{c}\text { Porcentajes } \\
\text { relativos }\end{array}$ & $\begin{array}{c}\text { Porcentajes } \\
\text { acumulados }\end{array}$ \\
\hline 4 & 7 & 46.6 & 46.6 \\
3 & 4 & 26.7 & 73.3 \\
2 & 4 & 26.7 & 100 \\
\hline Total & 15 & 100 & \\
\hline
\end{tabular}

Según la tabla 7 , el puntaje que más se repitió fue 4 . Con respecto a la media, el $50 \%$ de los agricultores entrevistados obtuvo un puntaje por encima de 3 y el $50 \%$ restante obtuvo un puntaje por debajo de este valor.

Los agricultores de Jesús del Valle que han participado en la ECA poseen mayor información y conocimiento acerca del "transporte y almacenamiento de plaguicidas" en un nivel de confianza del 95\%, que los agricultores que no participaron.

Según las Fig. 1 y 2, los agricultores que participaron de la ECA sumaron un puntaje total de $62(82.6 \%)$ en el tema de "preparación de la mezcla y equipos" mientras que los que no participaron un total de 47 (62.6\%). En promedio se puede ver que los participantes de la ECA obtuvieron 4.13 puntos, mientras que los no participantes a la ECA obtuvieron un promedio de 3.13 puntos. Los agricultores que participaron en la ECA ahora poseen un $20 \%$ más de conocimiento acerca de la "preparación de la mezcla y equipos" para hacer las aplicaciones, respecto a los no participantes. Ver tabla 8.

Para evaluar si existe una diferencia significativa entre los puntajes de agricultores que asistieron a la ECA y los que no lo hicieron respecto a "preparación de la mezcla y equipos", se aplicó la prueba "t" arrojando los siguientes resultados:
Tabla 8. Puntaje total, promedio y porcentaje de acierto en el tema "preparación de la mezcla y equipos", entre agricultores participantes y no participantes en la ECA.

\begin{tabular}{cccc}
\hline & $\begin{array}{c}\text { Total } \\
\text { puntaje }\end{array}$ & Promedio & $\begin{array}{c}\% \\
\text { Acierto }\end{array}$ \\
\hline $\begin{array}{c}\text { Participantes de la } \\
\text { ECA }\end{array}$ & 62 & 4.13 & 82.6 \\
$\begin{array}{c}\text { No participantes de } \\
\text { la ECA }\end{array}$ & 47 & 3.13 & 62.6 \\
\hline
\end{tabular}

Tabla 9. Puntajes registrados para los participantes de la ECA en el tema "preparación de la mezcla y equipos".

\begin{tabular}{cccc}
\hline $\begin{array}{c}\text { Puntajes } \\
\text { registrados }\end{array}$ & Frecuencias & $\begin{array}{c}\text { Porcentajes } \\
\text { relativos }\end{array}$ & $\begin{array}{c}\text { Porcentajes } \\
\text { acumulados }\end{array}$ \\
\hline 5 & 6 & 40 & 40 \\
4 & 5 & 33.3 & 73.3 \\
3 & 4 & 26.7 & 100 \\
\hline Total & 15 & 100 & \\
\hline
\end{tabular}

Según la tabla 9 , el puntaje que más se repitió fue 5 . Con respecto a la media, el $50 \%$ de los agricultores entrevistados obtuvo un puntaje por encima de 4 y el 50\% restante obtuvo un puntaje por debajo de este valor. En promedio, el puntaje obtenido es de 4.13. Asimismo, se desvía de 4.13 (en promedio) 0.81 puntos.

Tabla 10. Puntajes registrados para los no participantes de la ECA en el tema "preparación de la mezcla y equipos".

\begin{tabular}{cccc}
\hline $\begin{array}{c}\text { Puntajes } \\
\text { registrados }\end{array}$ & Frecuencias & $\begin{array}{c}\text { Porcentajes } \\
\text { relativos }\end{array}$ & $\begin{array}{c}\text { Porcentajes } \\
\text { acumulados }\end{array}$ \\
\hline 4 & 7 & 46.7 & 46.7 \\
3 & 3 & 20 & 66.7 \\
2 & 5 & 33.3 & 100 \\
\hline Total & 15 & 100 & \\
\hline
\end{tabular}

Según la tabla 10, el puntaje que más se repitió fue 4 . Con respecto a la media, el $50 \%$ de los agricultores entrevistados obtuvo un puntaje por encima de 3 y el $50 \%$ restante obtuvo un puntaje por debajo de este valor. Los agricultores participaron en la ECA poseen mayor información y conocimiento acerca de la "preparación de la mezcla y equipos" en un nivel de confianza del $99 \%$, que los agricultores que no participaron. Según las Fig. 1 y 2, los agricultores que participaron en la ECA sumaron un puntaje total de 65 (86.6) en el tema de "protección durante la aplicación", mientras que los que no participaron un total de $36(48 \%)$. En promedio se puede ver que los participantes de la ECA obtuvieron 4.33 puntos, mientras que los no participantes de la ECA obtuvieron un promedio de 2.4 puntos.

Es decir, los agricultores que participaron en la ECA ahora poseen un $38.6 \%$ más de conocimiento acerca de la "protección durante la aplicación". Ver tabla 11. 
Tabla 11. Puntaje total, promedio y porcentaje de acierto en el tema "protección durante la aplicación", entre agricultores participantes y no participantes en la ECA.

\begin{tabular}{cccc}
\hline & $\begin{array}{c}\text { Total } \\
\text { puntaje }\end{array}$ & Promedio & $\begin{array}{c}\% \\
\text { Acierto }\end{array}$ \\
\hline Participante de la ECA & 65 & 4.33 & 86.6 \\
No participantes de la ECA & 36 & 2.4 & 48 \\
\hline
\end{tabular}

Para evaluar si existe una diferencia significativa entre los puntajes de agricultores que participaron en la ECA y los que no lo hicieron respecto a la "protección durante la aplicación", se aplicó la prueba "t" arrojando los siguientes resultados:

Tabla 12. Puntajes registrados para los participantes de la ECA en el tema "protección durante la aplicación".

\begin{tabular}{cccc}
\hline $\begin{array}{c}\text { Puntajes } \\
\text { registrados }\end{array}$ & Frecuencias & $\begin{array}{c}\text { Porcentajes } \\
\text { relativos }\end{array}$ & $\begin{array}{c}\text { Porcentajes } \\
\text { acumulados }\end{array}$ \\
\hline 5 & 8 & 53.3 & 53.3 \\
4 & 4 & 26.7 & 80 \\
3 & 3 & 20 & 100 \\
\hline Total & 15 & 100 & \\
\hline
\end{tabular}

Según la tabla 12 , el puntaje que más se repitió fue 5 . Con respecto a la media, el $50 \%$ de los agricultores entrevistados obtuvo un puntaje por encima de 5 y el $50 \%$ restante obtuvo un puntaje por debajo de este valor. En promedio, el puntaje obtenido es de 4.33. Asimismo, se desvía de 4.33 (en promedio) 0.79 puntos.

Tabla 13. Puntajes registrados para los no participantes de la ECA en el tema "protección durante la aplicación".

\begin{tabular}{cccc}
\hline $\begin{array}{c}\text { Puntajes } \\
\text { registrados }\end{array}$ & Frecuencias & $\begin{array}{c}\text { Porcentajes } \\
\text { relativos }\end{array}$ & $\begin{array}{c}\text { Porcentajes } \\
\text { acumulados }\end{array}$ \\
\hline 4 & 3 & 20 & 20 \\
3 & 3 & 20 & 40 \\
2 & 6 & 40 & 80 \\
1 & 3 & 20 & 100 \\
\hline Total & 15 & 100 & \\
\hline
\end{tabular}

Según la tabla 13, el puntaje que más se repitió fue 2 . Con respecto a la media, el $50 \%$ de los agricultores entrevistados obtuvo un puntaje por encima de 2 y el $50 \%$ restante obtuvo un puntaje por debajo de este valor.

Los agricultores que participaron de la ECA poseen mayor información y conocimiento acerca de la "protección durante la aplicación" en un nivel de confianza del 99\%, que los agricultores que no participaron.

Según las Fig. 1 y 2, los agricultores que participaron de la ECA sumaron un puntaje total de $60(80 \%)$ en el tema de post aplicación, mientras que los que no participaron un total de 49 (65.4\%). En promedio, los participantes de la ECA obtuvieron 4 puntos, y los no participantes obtuvieron un promedio de 3.27 puntos. Es decir, los agricultores que participaron en la ECA ahora poseen un $14.6 \%$ más de conocimiento acerca de las medidas de "post-aplicación de plaguicidas", respecto a los no participantes. (Tabla 14).

Tabla 14. Puntaje total, promedio y porcentaje de acierto en el tema "post aplicación", entre agricultores participantes y no participantes en la ECA.

\begin{tabular}{lccc}
\hline & $\begin{array}{c}\text { Total } \\
\text { puntaje }\end{array}$ & Promedio & $\begin{array}{c}\% \\
\text { Acierto }\end{array}$ \\
\hline Participantes de la ECA & 60 & 4 & 80 \\
No participantes de la ECA & 49 & 3.27 & 65.4 \\
\hline
\end{tabular}

Para evaluar si existe una diferencia significativa entre los puntajes de agricultores que participaron en la ECA y los que no lo hicieron respecto a la "post-aplicación", se aplicó la prueba " $t$ ” arrojando los siguientes resultados:

Tabla 15. Puntajes registrados para los participantes de la ECA en el tema "post-aplicación".

\begin{tabular}{cccc}
\hline $\begin{array}{c}\text { Puntajes } \\
\text { registrados }\end{array}$ & Frecuencias & $\begin{array}{c}\text { Porcentajes } \\
\text { relativos }\end{array}$ & $\begin{array}{c}\text { Porcentajes } \\
\text { acumulados }\end{array}$ \\
\hline 5 & 5 & 33.3 & 33.3 \\
4 & 6 & 40 & 73.3 \\
3 & 3 & 20 & 93.3 \\
2 & 1 & 6.7 & 100 \\
\hline Total & 15 & 100 & \\
\hline
\end{tabular}

Según la tabla 15, el puntaje que más se repitió fue 4 . Con respecto a la media, el $50 \%$ de los agricultores entrevistados obtuvo un puntaje por encima de 4 y el $50 \%$ restante obtuvo un puntaje por debajo de este valor. En promedio, el puntaje obtenido es de 4. Asimismo, se desvía de 4 (en promedio) 0.89 puntos.

Tabla 16. Puntajes registrados para los no participantes de la ECA en el tema "post-aplicación”.

\begin{tabular}{cccc}
\hline $\begin{array}{c}\text { Puntajes } \\
\text { registrados }\end{array}$ & Frecuencias & $\begin{array}{c}\text { Porcentajes } \\
\text { relativos }\end{array}$ & $\begin{array}{c}\text { Porcentajes } \\
\text { acumulados }\end{array}$ \\
\hline 5 & 2 & 13.3 & 13.3 \\
4 & 2 & 13.3 & 26.6 \\
3 & 9 & 60 & 86.6 \\
2 & 2 & 13.4 & 100 \\
\hline Total & 15 & 100 & \\
\hline
\end{tabular}

Según la tabla 20, el puntaje que más se repitió fue 3 . Con respecto a la media, el $50 \%$ de los agricultores entrevistados obtuvo un puntaje por encima de 3 y el $50 \%$ restante obtuvo un puntaje por debajo de este valor. Los agricultores de Jesús del Valle que han participado en la 
ECA poseen mayor información y conocimiento acerca de la "post-aplicación" en un nivel de confianza del 95\%, que los agricultores que no participaron.

En cuanto a la decisión de usar plaguicidas, en una ECA se enseña a usar plaguicidas sólo después de realizar una evaluación en campo de las plagas y los insectos benéficos. El conocimiento adquirido mediante la ECA sobre este tema influye en el $40 \%$ de los encuestados al momento de decidir usar un plaguicida. Sin embargo, es más fuerte la influencia de las recomendaciones de los técnicos de tiendas ya que más de la mitad (53.3\%) de ellos se basan en estas para decidir usar un plaguicida. En el caso de los agricultores que no han pasado por una ECA es más fuerte aún la importancia de las recomendaciones de los técnicos al momento de decidir usar un plaguicida (un $66.7 \%$ las usan como referencia). Incluso le dan más énfasis a su costumbre de hacer aplicaciones calendarizadas $(20 \%)$ que a una evaluación previa del campo (13.3\%). Ver tabla 17.

Estos resultados son semejantes a los hallados en el estudio de Rodríguez (2006) donde se entrevistó a una muestra de agricultores en general de la provincia de Huaral. Se aprecia que un buen número de agricultores (48.7\%) decide usar plaguicidas de acuerdo con las recomendaciones de los técnicos, seguido de la evaluación de campo que sólo la considera el $26.3 \%$ de la muestra. Rodríguez (2003), encontró que el 54.3\% de agricultores de Barranca, sin haber participado en alguna capacitación, cuando tenía algún problema en campo buscaba la ayuda en una casa comercial; y entre la empresa o institución que promociona técnicas agrícolas, un 40.4\% señaló a la casas comerciales y tiendas locales y un $15.89 \%$ mencionaron a empresas privadas de agroquímicos.

En cambio, en el estudio realizado por Groeneweg, Versteeg y Chavez Tafur (FAO-2004) que evaluó agricultores que asistieron a varias ECAs en diferentes departamentos, arroja que lo que más influye (más del $70 \%$ de los encuestados) en su decisión es la evaluación previa del campo. Sólo un 10\% sigue las recomendaciones de los técnicos.

Tabla 17. Fuentes de información y su influencia en la toma de decisiones de aplicación y uso de plaguicidas entre los agricultores de Jesús del Valle.

\begin{tabular}{lcccc}
\hline & \multicolumn{2}{c}{ Asistente a la ECA } & \multicolumn{2}{c}{$\begin{array}{c}\text { No asistente a la } \\
\text { ECA }\end{array}$} \\
\cline { 2 - 5 } & Frecuencia & $\begin{array}{c}\% \\
\text { relativo }\end{array}$ & Frecuencia & $\begin{array}{c}\% \\
\text { relativo }\end{array}$ \\
\hline $\begin{array}{l}\text { Por la evaluación } \\
\text { de campo }\end{array}$ & 6 & 40 & 2 & 13.3 \\
$\begin{array}{l}\text { Por } \\
\text { recomendación } \\
\text { del técnico }\end{array}$ & 8 & 53.3 & 10 & 66.7 \\
Por costumbre & 1 & 6.7 & 3 & 20 \\
\hline \multicolumn{1}{c}{ Total } & 15 & 100 & 15 & 100 \\
\hline
\end{tabular}

\section{Referencias Bibliográficas}

Barrera, V.; Escudero, L.; Alwang, J. y Norton, G. 2003. Encontrando salidas para reducir los costos y la exposición a plaguicidas: experiencias con ECAs en el norte de Ecuador, en Leisa: Revista de Agroecología. Volumen $19 \mathrm{~N}^{\circ} 1: 46-48$.

Cáceres, O.; López, J. y Rueda, A. 2003. "Empoderamiento de agricultores para incentivar la producción y reducir plaguicidas en Centro América, en Leisa: Revista de Agroecología. Volumen 19 N¹: 49 52. 
Groeneweg, K.; Versteeg, A. y Chavez, J. 2004. Más nos han enseñado, mucho hemos aprendido. Proyecto FAO / GCP / PER / 036 / NET, Lima, Perú. 108 p.

Hernández, S. R.; Fernández, C., y Baptista, L., P. 1998. Metodología de la Investigación. McGraw Hill Interamericana. México. 705 p.

Ortiz, O.; Orrego, R., y Ho Raúl. 2003. Colaboración para la innovación tecnológica y metodológica: la experiencia del CIP y CARE con las ECAs, en Leisa: Revista de Agroecología. Volumen 19 N¹: 20 - 21.

PROTEC. 2007. Informe Técnico: Programa de Capacitación del PROTEC en Uso y Manejo Correcto de Agroquímicos 2005-2007. Lima, Perú. 30 p.

Rodríguez, SP. 2003. Posicionamiento de la extensión agrícola entre los agricultores de Barranca. Tesis Mag. Sc. Escuela de Post Grado de la Universidad Nacional Agraria La Molina. Lima, Perú. 133 p.

Rodríguez, SP. 2006. Informe final de Resultados: Riesgos en el uso y manejo de plaguicidas entre los agricultores de Huaral. PROTEC. Lima, Perú. 29 p. 